\title{
SWIFT X-RAY TELESCOPE AND VERY LARGE TELESCOPE OBSERVATIONS OF THE AFTERGLOW OF GRB 041223
}

\author{
D. N. Burrows, ${ }^{1}$ J. E. Hill, ${ }^{1}$ G. Chincarini, ${ }^{2,3}$ G. Tagliaferri, ${ }^{2}$ S. Campana, ${ }^{2}$ A. Moretti, ${ }^{2}$ P. Romano, ${ }^{2}$ D. Malesani, ${ }^{4}$ \\ J. L. Racusin, ${ }^{1}$ S. Kobayashi, ${ }^{1,5}$ B. Zhang, ${ }^{6}$ P. Mészáros, ${ }^{1}$ P. T. O’Brien, ${ }^{7}$ R. Willingale, ${ }^{7}$ J. P. Osborne, ${ }^{7}$ \\ G. Cusumano, ${ }^{8}$ P. Giommi, ${ }^{9}$ L. Angelini, ${ }^{10,11}$ A. F. Abbey, ${ }^{12}$ L. A. Antonelli, ${ }^{13}$ A. P. Beardmore, ${ }^{7}$ \\ M. Capalbi, ${ }^{9}$ S. Covino, ${ }^{2}$ P. D’Avanzo, ${ }^{3}$ M. R. Goad,${ }^{7}$ J. A. Kennea, ${ }^{1}$ D. C. Morris, ${ }^{1}$ C. Pagani, ${ }^{1,2}$ \\ K. L. Page, ${ }^{7}$ L. Stella, ${ }^{13}$ J. A. Nousek, ${ }^{1}$ A. A. Wells, ${ }^{12}$ and N. Gehrels ${ }^{10}$ \\ Received 2005 January 21; accepted 2005 February 15; published 2005 March 9
}

\begin{abstract}
GRB 041223 was the first gamma-ray burst detected by the Swift X-Ray Telescope (XRT), beginning $4.6 \mathrm{hr}$ after the burst. The X-ray detection triggered a VLT observation of the optical/near-infrared (NIR) counterpart, located about 1".1 from the XRT position. The X-ray counterpart faded rapidly, with a power-law index of $-1.72 \pm 0.20$, with an average unabsorbed X-ray flux of $6.5 \times 10^{-12} \mathrm{ergs} \mathrm{cm}^{-2} \mathrm{~s}^{-1}$ in the $0.5-10 \mathrm{keV}$ band for a power-law spectrum of photon index $2.02 \pm 0.13$ with Galactic absorption. The NIR counterpart was observed at three epochs between 16 and $87 \mathrm{hr}$ after the burst and faded with a power-law index of $-1.14 \pm 0.08$ with a reddening-corrected spectral energy distribution power-law slope of $-0.40 \pm 0.03$. We find that the X-ray and NIR data are consistent with a two-component jet in a wind medium.
\end{abstract}

Subject heading: gamma rays: bursts

Online material: color figures

\section{INTRODUCTION}

The Swift gamma-ray burst mission (Gehrels et al. 2004) was launched on 2004 November 20 to discover and study gammaray bursts (GRBs). For over 25 years following their discovery by the Vela satellites (Klebesadel et al. 1973), GRBs remained one of the greatest mysteries in astrophysics, largely because of a frustrating inability to pinpoint their locations on the sky and to detect any counterparts at other wavelengths. This situation changed dramatically in 1997 February, when the BeppoSAX satellite discovered the first X-ray afterglow of a GRB (Costa et al. 1997), confirming the predictions of Mészáros \& Rees (1997) that X-ray, optical, and radio afterglows are produced by the expanding relativistic fireball that produces the burst itself. By the end of 2004, BeppoSAX, the Rossi X-Ray Timing Explorer, ASCA, Chandra, and XMM-Newton had found 55 X-ray afterglows, ${ }^{14}$ with observations typically beginning 6-24 hr after

\footnotetext{
${ }^{1}$ Department of Astronomy and Astrophysics, Pennsylvania State University, 525 Davey Laboratory, University Park, PA 16802; burrows@astro.psu.edu.

${ }^{2}$ INAF-Osservatorio Astronomico di Brera, Via Bianchi 46, 23807 Merate, Italy.

${ }^{3}$ Dipartimento di Fisica, Università degli studi di Milano-Bicocca, Piazza delle Scienze 3, 20126 Milan, Italy.

${ }^{4}$ International School for Advanced Studies, SISSA-ISAS, via Beirut 2-4, 34014 Trieste, Italy.

${ }^{5}$ Center for Gravitational Wave Physics, Pennsylvania State University, University Park, PA 16802.

${ }^{6}$ Department of Physics, University of Nevada, Box 454002, Las Vegas, NV 89154-4002.

${ }^{7}$ Department of Physics and Astronomy, University of Leicester, University Road, Leicester LE1 7RH, UK.

${ }^{8}$ INAF-Istituto di Astrofisica Spaziale e Fisica Cosmica Sezione di Palermo, Via Ugo La Malfa 153, 90146 Palermo, Italy.

${ }^{9}$ ASI Science Data Center, via Galileo Galilei, 00044 Frascati, Italy.

${ }^{10}$ NASA/Goddard Space Flight Center, Greenbelt, MD 20771.

${ }^{11}$ Department of Physics and Astronomy, Johns Hopkins University, 3400 North Charles Street, Baltimore, MD 21218.

${ }^{12}$ Space Research Centre, University of Leicester, Leicester LE1 7RH, UK.

${ }^{13}$ INAF-Osservatorio Astronomico di Roma, via di Frascati 33, 00040 Monteporzio, Italy.

${ }^{14}$ See http://www.mpe.mpg.de/ jcg/grbgen.html.
}

the burst, during which time the afterglow emission had faded by several orders of magnitude. The Swift X-Ray Telescope (XRT) was designed to make very early observations of X-ray afterglows, beginning approximately a minute after the discovery of a GRB by the Swift Burst Alert Telescope (BAT).

We report on the first GRB afterglow discovered by the XRT, on 2004 December 23, and on subsequent follow-up observations in the optical and near-infrared (NIR) bands with the VLT. GRB 041223 was discovered by the Swift BAT instrument at 14:06:18 UT on 2004 December 23 (Tueller et al. 2004). The burst lasted a total of $130 \mathrm{~s}$, with most of the emission occurring in a $60 \mathrm{~s}$ interval around the main peak. The peak brightness was about $3 \times 10^{-6} \mathrm{ergs} \mathrm{cm}^{-2} \mathrm{~s}^{-1}$ in the $15-350 \mathrm{keV}$ band (Markwardt et al. 2004). The Swift observatory was still in its checkout and activation phase, and automated slewing had not yet been enabled. The spacecraft was commanded to slew to the position reported by the BAT about $4.6 \mathrm{hr}$ after the burst occurred, and XRT observations began immediately following target acquisition (Burrows et al. 2004b; Tagliaferri et al. 2004).

\section{XRT AND VLT OBSERVATIONS}

The XRT instrument is described in detail in Burrows et al. (2004a, 2005). The instrument is designed to obtain automated observations of newly discovered bursts. However, the XRT was collecting calibration/test data in Photon-Counting mode (similar to Timed Exposure mode on the Chandra ACIS instrument) under manual control when the slew began and continued in this same readout mode throughout the slew and the subsequent observation of the GRB field, which began at 18:43:59 UT. The GRB field was observed on three consecutive orbits, with the second and third observations scheduled autonomously on board. Details of the XRT observations are given in Table 1 .

As soon as the X-ray position was communicated via the GRB Coordinate Network, we initiated VLT observations as part of the Multiwavelength Italian Swift Team and International Co-Investigators (MISTICI) team to look for the NIR afterglow (Malesani et al. 2004a, 2004b). Observations were 
TABLE 1

XRT OBSERVATIONS OF GRB 041223

\begin{tabular}{lccc}
\hline \hline Observation & $\begin{array}{c}\text { Start Time }^{\mathrm{a}} \\
(\mathrm{UT})\end{array}$ & $\begin{array}{c}\text { Time since GRB } \\
(\mathrm{hr})\end{array}$ & $\begin{array}{c}\text { Duration } \\
(\mathrm{s})\end{array}$ \\
\hline $1 \ldots \ldots \ldots \ldots$ & $18: 43: 59$ & 4.63 & 1479.3 \\
$2 \ldots \ldots \ldots \ldots$ & $20: 16: 24$ & 6.17 & 1491.8 \\
$3 \ldots \ldots \ldots \ldots$ & $21: 50: 40$ & 7.74 & 546.6 \\
\hline
\end{tabular}

${ }^{\mathrm{a}}$ On 2004 December 23

carried out with the Infrared Spectrometer Array and Camera (ISAAC) installed on the VLT-UT1, starting on 2004 December 24 at 06:09 UT (16.1 hr after the GRB). No new objects were found in comparison with the Two Micron All Sky Survey (2MASS) catalog. However, the object discovered in the $R$ band by Berger et al. (2004) was immediately recognized in our frames, at a brightness below the 2MASS catalog limit. Multiband observations carried out on the following nights clearly showed that the object was fading, confirming its afterglow nature. $R$-band data were collected using the Focal Reducer/ Low Dispersion Spectrograph 2 (FORS2) installed on the VLTUT1. The observation log is reported in Table 2.

\section{DATA ANALYSIS}

The XRT data were processed by the Swift Data Center at NASA/Goddard Space Flight Center (GSFC) to level 1 data products (calibrated and quality-flagged event lists), which were then further processed by hand, using the XRTDAS software package produced by the Agenzia Spaziale Italiana (ASI) Science Data Center. We used XRT event grades 0-4, which provide the best combination of spectral resolution and detection efficiency. Because of the unusual data collection mode, we had to perform several nonstandard data selections to ensure that the data were all taken while the observatory was pointed accurately at the target and to remove some times with anomalously high background rates. The CCD temperature was between $-47.4^{\circ} \mathrm{C}$ and $-51.8^{\circ} \mathrm{C}$, about $50^{\circ}$ warmer than the design temperature owing to a failure in the active cooling system. As a result of this high temperature, a large number of hot and flickering pixels are present; these were eliminated by the XRTDAS FTOOL xrthotpix. The total exposure time after data screening was $3518 \mathrm{~s}$. The final $0.2-10 \mathrm{keV}$ image is shown in Figure 1.

The X-ray afterglow position determined using the X-ray image analysis tool ximage is R.A. (J2000.0) $=06^{\mathrm{h}} 40^{\mathrm{m}} 47.5$, decl. $(\mathrm{J} 2000.0)=-37^{\circ} 04^{\prime} 22^{\prime \prime}$.9. This position is indicated on Figure 2, along with the initial position given in Burrows et al. (2004b). We estimate a position uncertainty of $8^{\prime \prime}(90 \%$ confidence). This includes a systematic error of about $5^{\prime \prime}$ due to residual misalignment between the XRT and the star trackers, which is still being calibrated. The position determined by the XRT for this afterglow is $50^{\prime \prime}$ from the BAT position and only 1"1 from the position of the optical/NIR counterpart. The Xray afterglow designation is SWIFT J064047.5-370423.

The $0.5-10 \mathrm{keV}$ band light curve measured by the XRT is

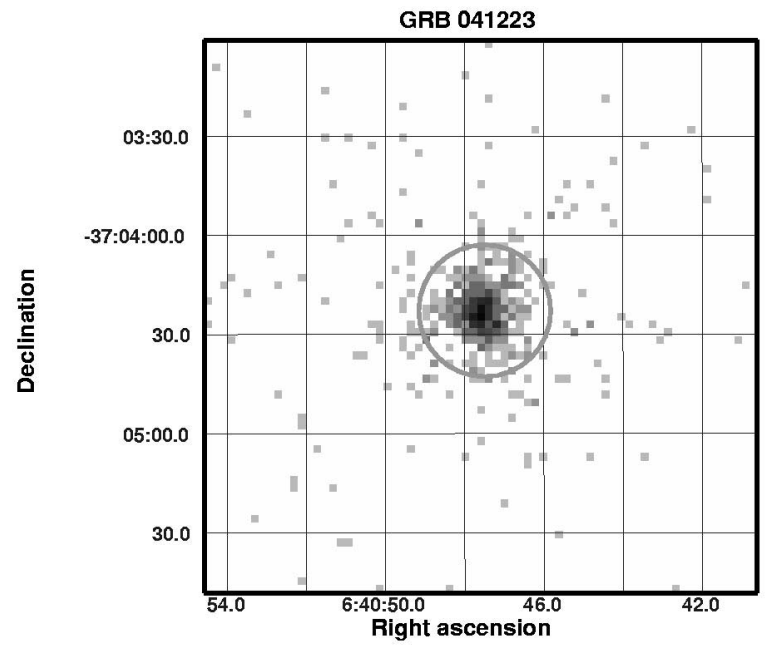

FIG. 1.-XRT image of the field of GRB $041223(0.5-10 \mathrm{keV}$, log intensity scale). The circle shows the region used for extraction of afterglow photons $\left(20^{\prime \prime}\right.$ radius). A total of $520 \mathrm{X}$-rays were detected from this afterglow after background subtraction. [See the electronic edition of the Journal for a color version of this figure.]

shown in Figure 3. The data were extracted from a $20^{\prime \prime}$ radius circle. The background measured in a circular region of 47.2 radius, located away from any hot or flickering pixels, was subtracted. The X-ray source is clearly fading with a powerlaw slope of $\alpha_{\mathrm{X}}=-1.72 \pm 0.20$ (68\% confidence level) for a decay law of the form $F_{v}(t) \propto t^{\alpha_{\nu}}$.

The XRT spectrum of the combined data set is shown in Figure 4. The XRT in-flight calibration had not yet begun, and the spectral fit was based on ground calibration data taken at the intended operating temperature of $-100^{\circ} \mathrm{C}$. The only modification to the prelaunch calibration parameters was a change of $2 \%$ in gain, based on preliminary analysis of our first-light observation of Cas A. The spectral results should therefore be treated with some caution until a reanalysis can be performed, following onorbit instrument calibration observations scheduled to begin in 2005 mid-January. However, preliminary spectral analysis of an XRT observation of the Crab Nebula yielded spectral parameters in reasonable agreement with recent measurements from Beppo SAX/MECS (G. Cusumano 2004, private communication), XMM-Newton/EPIC (Willingale et al. 2001), and Chandra/ACIS (Mori et al. 2004), providing some confidence in these preliminary spectral fits. We estimate that the systematic errors in spectral index and $N_{\mathrm{H}}$ are no more than $10 \%$. (The uncertainties quoted below do not include systematic errors.)

With these caveats, we were able to fit the backgroundsubtracted spectrum to an absorbed power law. The spectrum was processed with a minimum of 20 counts per bin, with channels below $0.2 \mathrm{keV}$ and above $10 \mathrm{keV}$ ignored. The spectral analysis used the same screening as the light curve and is based on 520 events, all of which were found below $\sim 7 \mathrm{keV}$.

TABLE 2

VLT OBSERVATIONS OF GRB 041223

\begin{tabular}{|c|c|c|c|c|c|c|}
\hline $\begin{array}{l}\text { Mean Date } \\
\text { (UT) }\end{array}$ & $\begin{array}{c}\text { Time since GRB } \\
(\mathrm{hr})\end{array}$ & Filter & $\begin{array}{l}\text { Exposure Time } \\
(\text { Exposures } \times s)\end{array}$ & $\begin{array}{l}\text { Seeing } \\
(\operatorname{arcsec})\end{array}$ & Instrument & Magnitude \\
\hline $2004 \operatorname{Dec} 24.261$ & 16.15 & $J$ & $12 \times 60$ & 0.56 & ISAAC & $19.51 \pm 0.05$ \\
\hline 2004 Dec 25.046 & 34.99 & $R$ & $2 \times 120$ & 0.38 & FORS2 & $21.60 \pm 0.04$ \\
\hline 2004 Dec 25.070 . & 35.57 & $J$ & $20 \times 60$ & 0.55 & ISAAC & $20.43 \pm 0.05$ \\
\hline 2004 Dec $25.089 \ldots \ldots$ & 36.05 & $K$ & $20 \times 60$ & 0.48 & ISAAC & $19.12 \pm 0.10$ \\
\hline 2004 Dec $27.213 \ldots \ldots$ & 87.0 & $J$ & $20 \times 60$ & 0.55 & ISAAC & $21.72 \pm 0.15$ \\
\hline
\end{tabular}




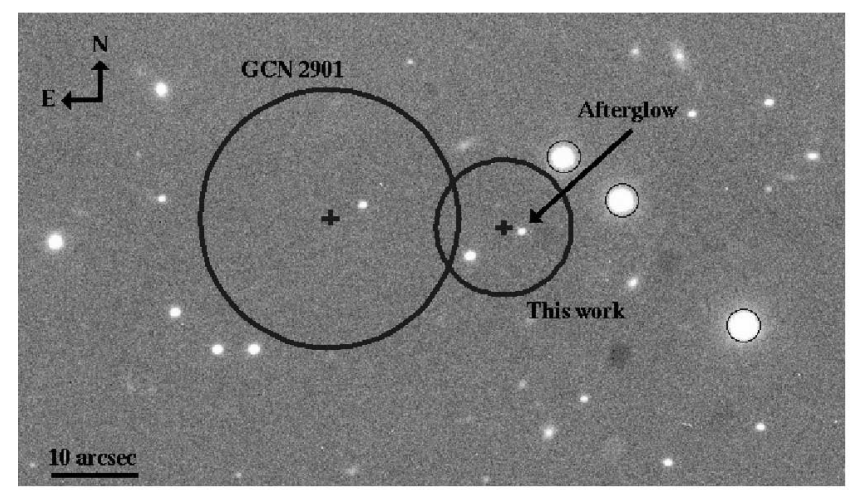

FIG. 2.- J-band image of the GRB 041223 afterglow. The error circles from the initial and final XRT positions are indicated. The final X-ray position is 1".1 from the NIR afterglow. The three circled stars were used for flux calibration of the J-band data. [See the electronic edition of the Journal for a color version of this figure.]

We fit the spectrum with a simple power law, yielding a good fit with $\chi^{2}=15.4$ for 22 degrees of freedom (providing a null hypothesis probability of 0.84 ). The fitted column density is $N_{\mathrm{H}}=1.5_{-0.5}^{+0.4} \times 10^{21} \mathrm{~cm}^{-2}$ (90\% confidence level), in good agreement with the Galactic value of $N_{\mathrm{H}} \sim 1.1 \times 10^{21} \mathrm{~cm}^{-2}$ (Dickey \& Lockman 1990). The power-law photon index is $\Gamma=$ $2.01_{-0.20}^{+0.23}$ (90\% confidence; the $68 \%$ confidence contours give $2.02 \pm 0.13$ for the photon index). The $0.5-10 \mathrm{keV}$ unabsorbed flux, averaged over the time of our observations, is $6.5 \times 10^{-12}$ ergs $\mathrm{cm}^{-2} \mathrm{~s}^{-1}$.

Our combined $J$-band image from 2004 December 24.261 is shown in Figure 2. The coordinates of the $J$-band afterglow are R.A. $(\mathrm{J} 2000.0)=06^{\mathrm{h}} 40^{\mathrm{m}} 47^{\mathrm{s}} .33$, decl. $(\mathrm{J} 2000.0)=$ $-37^{\circ} 04^{\prime} 23^{\prime \prime}$. 14 , with an estimated error of $0{ }^{\prime \prime} 16$, consistent with those reported by Berger et al. (2004). Absolute photometric calibration was performed against three bright, nonsaturated stars from the 2MASS catalog (circled in Fig. 2). The $J$-band afterglow has magnitude $J=19.51 \pm 0.05$ (Table 2). This object had faded to $J=20.43 \pm 0.05$ by 01:40 UT on 2004 December 25 , clearly marking it as the GRB afterglow. In our latest image (3.6 days after the GRB), the object is still pointlike at the resolution of the VLT images (0".55), with no signs of diffuse emission. The $J$-band decay index is $\alpha_{J}=-1.14 \pm 0.08$ (16-87 hr after the burst).

We also obtained $K$-band and $R$-band observations on 2004 December 25 . The $R$-band data were calibrated by observing the standard field PG $0231+051$. We used the $J$-band decay law to extrapolate the NIR data to a common epoch (1.50 days after the GRB). The photometric spectral energy distribution for these data is very well reproduced with a hard power law of spectral index $\beta_{\mathrm{NIR}}=-0.40 \pm 0.03$ for $F_{\nu} \propto \nu^{\beta}$, after correcting for Galactic extinction of $A_{V}=0.394$ mag (Schlegel et al. 1998)

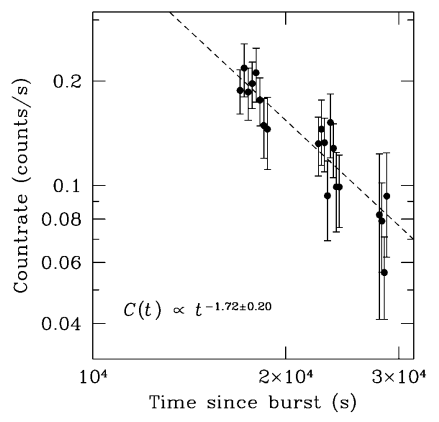

FIG. 3.-X-ray light curve of the afterglow of GRB 041223.

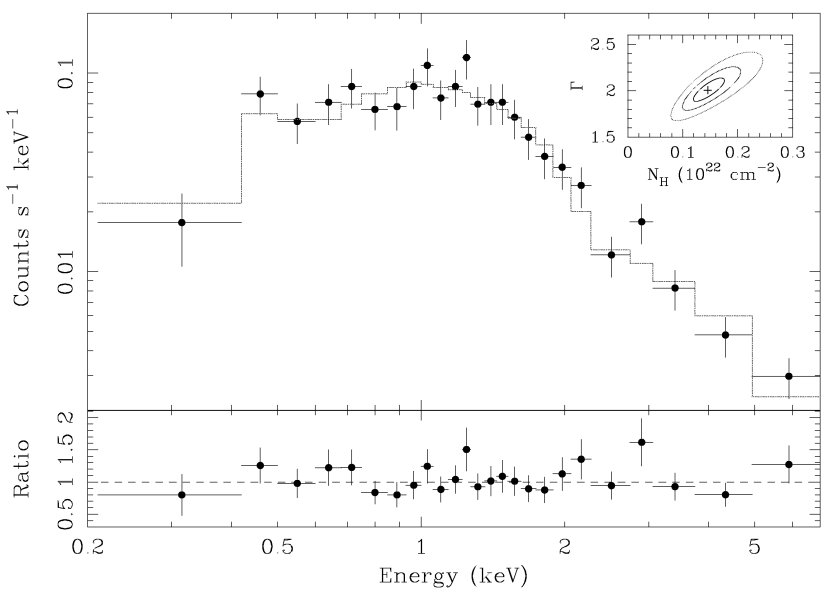

FIG. 4.-X-ray spectrum of the afterglow of GRB 041223, with the bestfit absorbed power-law model. The inset shows $1 \sigma, 2 \sigma$, and $3 \sigma$ confidence contours for the photon index and column density. [See the electronic edition of the Journal for a color version of this figure.]

and assuming a $10 \%$ uncertainty in the extinction. Such hard values are not common for afterglows at these stages (even without any reddening correction, the spectrum is still hard, with $\left.\beta_{\mathrm{NIR}}=-0.65\right)$.

\section{DISCUSSION AND CONCLUSIONS}

The observed decay and spectral indices are summarized in Table 3. The X-ray properties of the afterglow are similar to other X-ray afterglows in the literature. For example, the X-ray decay index, $\alpha_{\mathrm{X}}=-1.72$, is steeper than average but consistent with the distribution of BeppoSAX afterglows (Piro 2004). The NIR light curve is much flatter. These data cannot be explained in terms of any of the standard afterglow models summarized in Table 1 of Zhang \& Mészáros (2004). Fast cooling models do not fit the observed values of $\alpha$ and $\beta$ and are ruled out for this afterglow. Slow cooling models have $\beta>0$ for frequencies below the synchrotron injection frequency, $\nu_{m}$, which is also inconsistent with our data. The spectral break between the NIR and X-ray bands then requires $\nu_{m}<\nu_{\mathrm{NIR}}<\nu_{c}<\nu_{\mathrm{X}}$. Table 4 shows the remaining cases for three different afterglow models, in which $\alpha$ and $\beta$ are functions of the electron index, $p$. For each band, we give the power-law index of the underlying electron distribution, $p_{\beta}$, derived from the spectral index, $\beta$. We then calculate the value of the electron index, $p_{\alpha}$, derived from the temporal index, $\alpha$, using the relationship ${ }^{15}$ applicable to the observationally determined value of $p_{\beta}$ (Zhang \& Mészáros 2004). Finally, we give the least-squares weighted average, $\langle p\rangle$, of $p_{\alpha}$ and $p_{\beta}$; its uncertainty (for $\chi_{\min }^{2}+1.0$ ); the minimum $\chi^{2}$; and the corresponding probability that the spectral and decay indices both arise from the same electron distribution. We find that no single

\footnotetext{
${ }^{15}$ In cases where $p_{\beta}<2$, we use the relations for $\alpha(p)$ derived by Dai \& Cheng (2001); were we instead to use the formulae applicable to $p>2,\langle p\rangle$ would change slightly but our conclusions would not be affected.
}

TABLE 3

Observed Decay and Spectral Power-Law Indices for GRB 041223

\begin{tabular}{lccc}
\hline \hline Band & Decay Index, $\alpha^{\mathrm{a}}$ & Spectral Index, $\beta^{\mathrm{a}}$ & Hours Postburst \\
\hline$J$ band $\ldots \ldots \ldots$. & $-1.14 \pm 0.08^{\mathrm{b}}$ & $-0.40 \pm 0.03^{\mathrm{b}}$ & $16-87$ \\
$0.5-10 \mathrm{keV} \ldots \ldots$ & $-1.72 \pm 0.20^{\mathrm{b}}$ & $-1.02 \pm 0.13^{\mathrm{b}}$ & $4.6-7.9$ \\
\hline${ }^{\mathrm{a}} F(t, \nu) \propto t^{\alpha} \nu^{\beta}$. & & & \\
${ }^{\mathrm{b}} 68 \%$ confidence limits. &
\end{tabular}


TABLE 4

Afterglow Model Fits to the Data

\begin{tabular}{|c|c|c|c|c|c|}
\hline Band & $p_{\beta}$ & $p_{\alpha}$ & $\langle p\rangle^{\mathrm{a}}$ & $\chi_{\min }^{2}$ & $P$ \\
\hline \multicolumn{6}{|c|}{ ISM, Slow Cooling Model } \\
\hline $\begin{array}{l}\mathrm{NIR}\left(\nu_{m}<\nu<\nu_{c}\right) \ldots \ldots \\
\mathrm{X} \text {-ray }\left(\nu>\nu_{c}\right) \ldots \ldots \ldots\end{array}$ & $\begin{array}{l}-2 \beta_{\mathrm{NIR}}+1=1.80 \pm 0.06 \\
-2 \beta_{\mathrm{X}}=2.04 \pm 0.26\end{array}$ & $\begin{array}{l}-(1 / 3)\left(16 \alpha_{J}+6\right)=4.08 \pm 0.43 \\
-(1 / 3)\left(4 \alpha_{\mathrm{X}}-2\right)=2.96 \pm 0.27\end{array}$ & $\begin{array}{l}1.84 \pm 0.06 \\
2.48 \pm 0.19\end{array}$ & $\begin{array}{r}27.6 \\
6.02\end{array}$ & $\begin{array}{l}1.9 \times 10^{-7} \\
0.014\end{array}$ \\
\hline \multicolumn{6}{|c|}{ Wind, Slow Cooling Model } \\
\hline $\begin{array}{l}\mathrm{NIR}\left(\nu_{m}<\nu<\nu_{c}\right) \ldots \ldots \\
\mathrm{X} \text {-ray }\left(\nu>\nu_{c}\right) \ldots \ldots \ldots\end{array}$ & $\begin{array}{l}-2 \beta_{\mathrm{NIR}}+1=1.80 \pm 0.06 \\
-2 \beta_{\mathrm{X}}=2.04 \pm 0.26\end{array}$ & $\begin{array}{l}-8\left(\alpha_{J}+1\right)=1.12 \pm 0.69 \\
-(1 / 3)\left(4 \alpha_{\mathrm{x}}-2\right)=2.96 \pm 0.27\end{array}$ & $\begin{array}{l}1.79 \pm 0.06 \\
2.48 \pm 0.19\end{array}$ & $\begin{array}{l}0.96 \\
6.02\end{array}$ & $\begin{array}{l}0.33 \\
0.014\end{array}$ \\
\hline \multicolumn{6}{|c|}{ Jet, Slow Cooling Model } \\
\hline $\begin{array}{l}\mathrm{NIR}\left(\nu_{m}<\nu<\nu_{c}\right) \ldots \ldots \\
\mathrm{X} \text {-ray }\left(\nu>\nu_{c}\right) \ldots \ldots \ldots\end{array}$ & $\begin{array}{l}-2 \beta_{\mathrm{NIR}}+1=1.80 \pm 0.06 \\
-2 \beta_{\mathrm{x}}=2.04 \pm 0.26\end{array}$ & $\begin{array}{l}-4 \alpha_{J}-6=-1.44 \pm 0.32 \\
-\alpha_{\mathrm{x}}=1.72 \pm 0.20\end{array}$ & $\begin{array}{l}1.69 \pm 0.06 \\
1.84 \pm 0.16\end{array}$ & $\begin{array}{r}99 \\
0.95\end{array}$ & $\begin{array}{l}<10^{-9} \\
0.33\end{array}$ \\
\hline
\end{tabular}

${ }^{\text {a }} 68 \%$ confidence limits.

model is consistent with both the NIR and X-ray data: the NIR data are consistent only with a wind model, while the X-ray data are consistent only with a sideways-expanding jet model.

A more complex afterglow model is required to fully explain the data. Since the data in these bands are not contemporaneous, we consider the possibility of a more complex two-component jet structure similar to that proposed by Berger et al. (2003) for GRB 030329, with a narrow ultrarelativistic jet producing the early X-ray flux and a broader, mildly relativistic component producing the optical emission at later times; however, the jets in the case of GRB 041223 appear to be expanding into a wind medium instead of a uniform density interstellar medium (ISM).

The steep X-ray light curve suggests that the X-ray jet break occurred before the XRT observation (i.e., less than $4.6 \mathrm{hr}$ after the burst), which implies that the X-ray flux is dominated by a highly collimated jet (compare with the jet break times and opening angles given in Fig. 1 and Table 1 of Frail et al. 2001, for example). The electron index derived from the X-ray data is $1.84 \pm 0.16$, somewhat less than, but consistent with, the range $p=2.0-2.5$ found for most GRB afterglows (Panaitescu \& Kumar 2002). We note that the jet break rollover timescale in a wind model is quite long, which could explain the shallowerthan-expected value for $\alpha_{\mathrm{X}}$ found at our mean time of $6 \mathrm{hr}$ postburst (Kumar \& Panaitescu 2000); if so, the true electron index may be closer to the value $p_{\beta}=2.04$, fully consistent with previous results.

The narrow component should also produce NIR emission, but we have no NIR data at this epoch to confirm this prediction. At later times, the NIR data are consistent with a broader jet expanding into a wind cavity, powered by the same underlying electron distribution. We postulate that this broad jet has a much smaller Lorentz factor than the narrow component and begins decelerating between 8 and $16 \mathrm{hr}$ postburst (in our frame). It therefore does not contribute to the earlier X-ray observation but dominates the later NIR observation in comparison with the rapidly fading narrow component.

This work is supported at Pennsylvania State University (PSU) by NASA contract NAS5-00136, at the University of Leicester (UL) by the Particle Physics and Astronomy Research Council on grant numbers PPA/G/S/00524 and PPA/Z/S/2003/00507, and at the Osservatorio Astronomico di Brera $(\mathrm{OAB})$ by funding from ASI on grant number I/R/039/04. We gratefully acknowledge the contributions of dozens of members of the XRT team at PSU, UL, OAB, GSFC, ASI Science Data Center, and our subcontractors, who helped make this instrument possible. This work is partly based on observations performed with ESO telescopes under program 074.D-0418. We thank the MISTICI collaboration for use of their VLT photometric data in this Letter, and we are grateful to the ESO staff at Paranal, in particular Olivier Marco and Jonathan Smoker, for carefully performing the VLT observations in service mode. Finally, we thank the referee for a prompt and helpful review of this Letter.

\section{REFERENCES}

Berger, E., Krzeminski, W., \& Hamuy, M. 2004, GCN Circ. 2902, http:// gcn.gsfc.nasa.gov/gcn/gcn3/2902.gcn3

Berger, E., et al. 2003, Nature, 426, 154

Burrows, D. N., et al. 2004a, Proc. SPIE, 5165, 201

. 2004b, GCN Circ. 2901, http://gcn.gsfc.nasa.gov/gen/gen3/2901.gen3 2005, Space Sci. Rev., in press

Costa, E., et al. 1997, Nature, 387, 783

Dai, Z. G., \& Cheng, K. S. 2001, ApJ, 558, L109

Dickey, J. M., \& Lockman, F. J. 1990, ARA\&A, 28, 215

Frail, D., et al. 2001, ApJ, 562, L55

Gehrels, N., et al. 2004, ApJ, 611, 1005

Klebesadel, R. W., Strong, I. B., \& Olson, R. A. 1973, ApJ, 182, L85

Kumar, P., \& Panaitescu, A. 2000, ApJ, 541, L9

Malesani, D., et al. 2004a, GCN Circ. 2903, http://gcn.gsfc.nasa.gov/gcn/gcn3/ 2903.gcn 3

— 2004b, GCN Circ. 2913, http://gen.gsfc.nasa.gov/gen/gen3/2913.gen3
Markwardt, C., et al. 2004, GCN Circ. 2909, http://gcn.gsfc.nasa.gov/gcn/gcn3/ 2909.gen 3

Mészáros, P., \& Rees, M. J. 1997, ApJ, 476, 232

Mori, K., Burrows, D. N., Hester, J. J., Pavlov, G. G., Shibata, S., \& Tsunemi, H. 2004, ApJ, 609, 186

Panaitescu, A., \& Kumar, P. 2002, ApJ, 571, 779

Piro, L. 2004, in ASP Conf Ser. 312, Third Rome Workshop on Gamma-Ray Bursts in the Afterglow Era, ed. M. Feroci et al. (San Francisco: ASP), 149

Schlegel, D. J., Finkbeiner, D. P., \& Davis, M. 1998, ApJ, 500, 525

Tagliaferri, G., et al. 2004, GCN Circ. 2910, http://gcn.gsfc.nasa.gov/gen/gcn3/ 2910.gcn 3

Tueller, J., et al. 2004, GCN Circ. 2898, http://gcn.gsfc.nasa.gov/gen/gcn3/ 2898.gen 3

Willingale, R., Aschenbach, B., Griffiths, R. G., Sembay, S., Warwick, R. S.,

Becker, W., Abbey, A. F., \& Bonnet-Bidaud, J.-M. 2001, A\&A, 365, L212

Zhang, B., \& Mészáros, P. 2004, Int. J. Mod. Phys. A, 19, 2385 